\title{
A monogenic Hasse-Arf theorem
}

\author{
par JAMES BORGER
}

\begin{abstract}
RÉsumÉ. On étend le théorème de Hasse-Arf de la classe des extensions résiduellement séparables des anneaux de valuation discrète complets à la classe des extensions monogènes.
\end{abstract}

AbsTRACT. I extend the Hasse-Arf theorem from residually separable extensions of complete discrete valuation rings to monogenic extensions.

Let $B / A$ be a finite extension of henselian discrete valuation rings which is generically Galois with group $G$, that is, for which the corresponding extension of fraction fields is Galois with group $G$. For $\sigma \in G-\{1\}$, let $I_{B}(\sigma)$ be the ideal of $B$ generated by $(\sigma-1) B$ and let $i_{B}(\sigma)$ be the length of the $B$-module $B / I_{B}(\sigma)$.

For any finite dimensional complex representation $\rho: G \rightarrow \operatorname{Aut}_{\mathbb{C}}(V)$, we define the naive Artin conductor exactly as we do when $B / A$ is residually separable, i.e., when the extension of residue fields is separable:

$$
\operatorname{ar}_{\mathrm{n}}(\rho)=e_{B / A}^{-1} \sum_{\sigma \neq 1}[\operatorname{dim}(V)-\operatorname{trace}(\rho(\sigma))] i_{B}(\sigma) .
$$

By looking at real parts, it is immediate that this is a non-negative rational number, and when $B / A$ is residually separable, the Hasse-Arf theorem $[3$, VI §2] tells us that it is also an integer.

In [4], De Smit shows that most of the classical ramification-theoretic properties of residually separable extensions $B / A$ hold in the slightly more general, "monogenic" case where we require only that $B$ is generated as an $A$-algebra by one element. The purpose of this note is to show that the Hasse-Arf theorem also holds in this context.

Partial results in this direction were obtained by Spriano [5]. A proof of the Hasse-Arf theorem in equal characteristic that is strong enough to cover monogenic extensions was outlined at the 1999 Luminy conference on ramification theory. It was based on a technical analysis of a refinement [2, 3.2.2] of Kato's refined Swan conductor [1], but since then, an elementary reduction to the classical Hasse-Arf theorem has been found.

The contents of this paper are contained in my dissertation (U.C. Berkeley, 2000), which was written under the direction of Hendrik Lenstra. 
Proposition 1. Let $B / A$ be a finite generically separable extension of henselian discrete valuation rings. Then the following are equivalent.

(i) There exists an $x \in B$ such that $B=A[x]$.

(ii) The second exterior power $\Omega_{B / A}^{2}$ of the module of relative Kähler differentials is zero.

(iii) There is a henselian discrete valuation ring $A^{\prime}$ that is finite over the maximal unramified subextension $A^{\mathrm{nr}}$ of $B / A$ such that $e_{A^{\prime}} / A^{\mathrm{nr}}=1$ and $B^{\prime} / A^{\prime}$ is a residually separable extension of discrete valuation rings, where $B^{\prime}=A^{\prime} \otimes_{A^{\mathrm{nr}}} B$.

Proof. De Smit $[4,4.2]$ shows that (i) follows from (ii). For any $A^{\prime}$ as in (iii), we have $B^{\prime} \otimes_{B} \Omega_{B / A}^{2} \cong B^{\prime} \otimes_{B} \Omega_{B / A^{\mathrm{nr}}}^{2} \cong \Omega_{B^{\prime} / A^{\prime}}^{2}=0$, so (iii) implies (ii). Now we show (i) implies (iii).

Assume, as we may, that $A=A^{\mathrm{nr}}$, and let $l / k$ denote the residue extension of $B / A$. Take some $x \in B$ such that $B=A[x]$ and let $\bar{x}$ denote the image of $x$ in $l$. Let $g(X) \in A[X]$ be a monic lift of the minimal polynomial $X^{q}-a$ of $\bar{x}$ over $k$. Since the maximal ideal of $B$ is generated by that of $A$ and $g(x)$, we may assume that $g(x)$ generates the maximal ideal of $B$. Then modulo the maximal ideal of $B$, we have $g(X+x) \equiv X^{q}+x^{q}-a \equiv X^{q}$, so $g(X+x)$ is an Eisenstein polynomial with coefficients in $B$. Now let $A^{\prime}$ be the discrete valuation ring $A[X] /(g(X))$. Then

$$
B^{\prime}=A^{\prime} \otimes_{A} B \cong B[X] /(g(X)) \cong B[X] /(g(X+x))
$$

is a discrete valuation ring which has the same residue field as $B$ and, hence, $A^{\prime}$.

Proposition 2. Let $B / A$ be a finite extension of henselian discrete valuation rings that is generically Galois with group $G$, and let $\rho: G \rightarrow \operatorname{Aut}_{\mathbb{C}}(V)$ be a finite dimensional representation of $G$. If $A^{\prime} / A$ is a finite extension of henselian discrete valuation rings such that $B^{\prime}=A^{\prime} \otimes_{A} B$ is a discrete valuation ring, then we have $\operatorname{ar}_{\mathrm{n}}\left(\rho^{\prime}\right)=e_{A^{\prime} / A} \operatorname{ar}_{\mathrm{n}}(\rho)$, where $\rho^{\prime}$ is $\rho$ viewed as a representation of the generic Galois group of the extension $B^{\prime} / A^{\prime}$.

Proof. For $\sigma \in G-\{1\}$, we have $I_{B^{\prime}}(\sigma)=A^{\prime} \otimes_{A} I_{B}(\sigma)=B^{\prime} \otimes_{B} I_{B}(\sigma)$, so

$$
\begin{aligned}
i_{B^{\prime}}(\sigma) & =\operatorname{length}_{B^{\prime}}\left(B^{\prime} / I_{B^{\prime}}(\sigma)\right)=\operatorname{length}_{B^{\prime}}\left(B^{\prime} \otimes_{B} B / I_{B}(\sigma)\right) \\
& =e_{B^{\prime} / B} \operatorname{length}_{B}\left(B / I_{B}(\sigma)\right)=e_{B^{\prime} / B} i_{B}(\sigma) .
\end{aligned}
$$

Thus

$$
\operatorname{ar}_{\mathrm{n}}\left(\rho^{\prime}\right)=e_{B^{\prime} / B} \frac{e_{B / A}}{e_{B^{\prime} / A^{\prime}}} \operatorname{ar}_{\mathrm{n}}(\rho)=e_{A^{\prime} / A^{\prime}} \operatorname{ar}_{\mathrm{n}}(\rho) .
$$


Corollary 3. Let $B / A$ be a finite monogenic extension of henselian discrete valuation rings that is generically Galois with group $G$, and let $\rho: G \rightarrow$ $\operatorname{Aut}_{\mathbb{C}}(V)$ be a finite dimensional representation of $G$. Then $\operatorname{ar}_{\mathrm{n}}(\rho)$ is an integer.

Proof. Restricting to the maximal unramified subextension of $B / A$ does not change the naive Artin conductor or the monogeneity of the extension. So assume $B / A$ is residually purely inseparable. Now just apply the previous proposition with $A^{\prime}$ taken as in the first proposition and then use the classical Hasse-Arf theorem.

Remark. One can define a naive Swan conductor $[1,6.7]$ as well. It also is an integer in the monogenic case but simply because it agrees with the naive Artin conductor whenever $B / A$ is monogenic and not residually separable. It is not, however, a good invariant even in the monogenic case: it is a consequence of results outlined at the Luminy conference that in the (monogenic) equal-characteristic case, the naive Swan conductor of a faithful, one-dimensional representation agrees with Kato's Swan conductor if and only if either $B / A$ is residually separable or $e_{B / A}=1$, whereas for general monogenic extensions in equal-characteristic, the naive Artin conductor of a one-dimensional representation is equal to a non-logarithmic, "Artin-type" variant of Kato's Swan conductor.

\section{References}

[1] K. KATo, Swan conductors for characters of degree one in the imperfect residue field case. Algebraic $K$-theory and algebraic number theory, ed. M. Stein and R. K. Dennis, Contemp. Math. 83, Amer. Math. Soc., Providence, 1989, 101-131.

[2] S. MAtsudA, On the Swan conductor in positive characteristic. Amer. J. Math. 119 (1997), no. 4, 705-739.

[3] J-P. Serre, Corps locaux. Deuxième édition. Hermann, Paris, 1968.

[4] B. DE SMIT, The Different and Differentials of Local Fields with Imperfect Residue Fields. Proc. Edin. Math. Soc. 40 (1997), 353-365.

[5] L. Spriano. Thesis, Université de Bordeaux, 1999.

James BORGER

The University of Chicago

Department of Mathematics

5734 University Avenue

Chicago, Illinois 60637-1546, USA

E-mail : borger@math.uchicago.edu 